\section{International Journal
and Food Technology}

\title{
Impact of Supplementation with Flax Seed Powder and Flax Seed Oil on the Lipid levels of Patients with Dyslipidemia
}

Deepika Sharma

*Corresponding Author: Deepika Sharma, E-mail: deepikasaraswat05@gmail.com

Citation: Deepika Sharma (2016) Impact of Supplementation with Flax Seed Powder and Flax Seed Oil on the Lipid levels of Patients with Dyslipidemia. Int J Nutr Sci \& Food Tech. 2:3, 89-94

Copyright: (C) 2016 Deepika Sharma, et al. This is an open-access article distributed under the terms of the Creative Commons Attribution License, which permits unrestricted use, distribution, and reproduction in any medium, provided the original author and source are credited

Received October 5, 2016; Accepted October 15, 2016; Published October 30, 2016.

Keywords: Dyslipidemia, Flax Seed Powder, Flax Seed Oil, Supplementation, Lipid Levels.

Cardiovascular diseases (CVD) are the most prevalent cause of death and disability in both developed as well as developing countries (Chaturvedi and Bhargava, 2007). According to National Commission on Macroeconomics and Health (NCMH), there would be around 62 million patients with CAD by 2015 in India and out of these 23 million would be patients younger than 40 years of age (Indrayan, 2006). Cardiovascular diseases are due to atherosclerosis of large and medium sized arteries and dyslipidemia has been found to be one of the most important contributing factors of CAD (NCEP-ATP III, 2001). Dyslipidemia describes a number of abnormality in lipoprotein homeostasis including elevated total cholesterol $(\geq 200 \mathrm{mg} / \mathrm{dl}$ ), low density lipoprotein cholesterol $(\geq 130 \mathrm{mg} / \mathrm{dl})$, triglycerides $(\geq 150 \mathrm{mg} / \mathrm{dl})$ and low levels of high density lipoprotein $(\leq 40 \mathrm{mg} / \mathrm{dl}$ ) (NCEP,ATP III, 2002). Alternative to hypolipidemic drugs, plant seeds and dietary supplements provided a potential source of lowering blood lipid profile and are widely used in several traditional systems of medicines. Flax seed (linseed, Linum Usitatissimum), an edible oil seed/grain and one of the oldest crops, was acknowledged as a functional food (Thompson, 2003). Flax seed has gained much attention because of its unique nutrient components and potential effect on the prevention of CVD (Bloedon, 2004).

The current chapter presents relevant review in the area of present research under the following headings:

- $\quad$ Prevalence of dyslipidemia

$>\quad$ Global

$>\quad$ India

- $\quad$ Chemical composition of flax seed

- Role of flax seeds powder in management of dyslipidemia
- $\quad$ Role of flax seed oil in management of dyslipidemia

Prevalence of Dyslipidemia

Global

Hypercholesterolemia and hypertension are important modifiable risk factors for cardiovascular disease. A study was conducted by Wong et al., 2006 in United States among 2,864 subjects from the National Health and Nutrition Examination Survey 2001 to 2002. The results indicated that the prevalence of hypercholesterolemia and hypertension was $47 \%$ and $30 \%$, respectively.

One another study was conducted by Margaret, 2009 among 3,810 United States adults, participating in the National Health and Nutrition Examination Survey 1999-2004. It was concluded from the results that the overall prevalence of dyslipidemia was $60.3 \%$.

Dyslipidemia has become one of the important health risk factor in the Chinese population. Zhao et al., 2007 had conducted a study to investigate the prevalence of dyslipidemia in the Chinese population. Plasma lipid profile was analyzed by using the data obtained during the Chinese National Nutrition and Health Survey (CNHS) in 2002 which involved 14,252 participants of age 18 years or older. The mean levels of total cholesterol (TC), triglycerides (TG) and high density lipoprotein cholesterol (HDL-C) were found to be $3.81 \mathrm{mmol} / \mathrm{L}, 1.10 \mathrm{mmol} / \mathrm{L}$ and $1.30 \mathrm{mmol} / \mathrm{L}$, respectively. The results indicated that the prevalence of hypercholesterolemia, hypertriglyceridemia and low HDL-cholesterol was $2.9 \%, 11.9 \%$ and $7.4 \%$, respectively.

Wong et al. (2011) also analyzed the prevalence of dyslipidemia among 2951 Chinese individuals and found that the prevalence of dyslipidemia was $56 \%$ and the factors likely associated with dyslipidemia were higher age, female gender, urban region, higher 
body mass index, higher income, higher diastolic blood pressure, higher blood concentration of glucose and smoking.

Hypercholesterolemia, hypertriglyceridemia and abnormally low high density lipoprotein cholesterol had increased considerably over the past 20 years in professional population in Beijing (Zhiyan et al., 2005). The study included 14,963 individuals ( 8572 males and 6391 females) of 20-90 years of age. It was concluded from the study that hypercholesterolemia was found in $6 \%$ of males and $2.8 \%$ of females in the younger age group (20-39 years) and in $20.2 \%$ of males and $38.7 \%$ of females in the older age group $(>60$ years). High density lipoprotein cholesterol was abnormally low in $7 \%$ of males and $1.6 \%$ of females. The increase was found to be higher in the middle age group (40-59 years) (Zhiyan et al., 2005).

The prevalence of dyslipidemia among Korean population of age $\geq$ 20 years increased from $32.4 \%$ in 1998 to $42.6 \%$ in 2001 and $44.1 \%$ in 2005. Compared with the Korea National Health and Nutrition Survey (KNHANES) in 1998, the prevalence of dyslipidemia was $47 \%$, higher in 2001 and $61 \%$ higher in 2005 . In 2005 , only $9.5 \%$ of people with dyslipidemia were aware of the disease, $5.2 \%$ used lipid lowering medication and $33.2 \%$ of patients with treatment, reached treatment goals. It was summarized from the data that the prevalence of dyslipidemia in Korea gradually increased between 1998 and 2005 (Lee et al., 2012).

A study by Chatlert et al. (2006) in Thailand demonstrated the effect of an urban life style on dyslipidemia by comparing the lipid profile. A total of 916 subjects (595 urban and 321 rural) between the age group 20 and 88 years were selected for the study. The results indicated that in both men and women the mean total cholesterol and low density lipoprotein cholesterol were significantly higher in urban v/s rural subjects. Total cholesterol values were $207 \mathrm{v} / \mathrm{s} 169 \mathrm{mg} / \mathrm{dl}$ for men and $204 \mathrm{v} / \mathrm{s} 192 \mathrm{mg} / \mathrm{dl}$ for women and low density lipoprotein cholesterol levels were $120 \mathrm{v} / \mathrm{s} 87 \mathrm{mg} / \mathrm{dl}$ for men and $122 \mathrm{v} / \mathrm{s} 110 \mathrm{mg} / \mathrm{dl}$ for women. The study demonstrated a significant difference between the urban and rural subject's lipid profile and the prevalence of dyslipidemia. Migration to urban centers and adopting an urban life style was related to the rising lipid profile and prevalence of dyslipidemia (Chartlert et al., 2006).

\section{India}

Hyperlipidemia and obesity have been traditionally considered as diseases of affluence. Mishra et al. (2001) had shown the results of a study that the prevalence of dyslipidemia was very high in urban slum population of northern India. A total of 532 subjects (170 males and 362 females) were included in the study. Hypercholesterolemia was observed in a high number in both males $(26.8 \%)$ and females $(27.5 \%)$ and similarly equally high number of subjects had high low density lipoprotein cholesterol levels. However hypertriglyceridemia was found to be more prevalent in males, although, the difference was not statistically significant. Further, low level of high density lipoprotein cholesterol was found to be $15.8 \%$ males and $16.7 \%$ female subjects (Mishra et al., 2001).
One another study was conducted in Mumbai, India among 1805 urban dwellers of age group $\geq 40$ years. It was summarized from the study that the prevalence of dyslipidemia was higher in males than in females. Among participants having total cholesterol concentration $\geq 200 \mathrm{mg} / \mathrm{dl}, 38.7 \%$ were males and $23.3 \%$ were females. High density lipoprotein cholesterol was abnormally low in $64.2 \%$ males and $33.8 \%$ in females. The prevalence of hypercholesterolemia and hyper-triglyceridemia was more prominent in 31-40 years of age group than in $\leq 30$ years of age group (Sawant et al., 2008).

Coronary heart disease is increasing in Indian subjects residing in urban areas and lipid abnormalities are important risk factors. Gupta et al. (2008) had conducted studies in western India in urban locations. The studies evaluated adults of $\geq 20$ years of age for multiple coronary risk factors (JHW-1- 1993-94; $\mathrm{n}=2212$, JHW2- 1999-2001; $\mathrm{n}=1123$, JHW-3- 2002-2003; $\mathrm{n}=458$ and JHW-42004-2005; $\mathrm{n}=1127)$. The data showed that the mean levels of various lipoproteins increased from JHW-1 to JHW-2 and then gradually in JHW-3 and JHW-4. Age adjusted mean values (mg/dl) in JHW-1, JHW-2, JHW-3 and JHW-4 studies showed a significant increase in total cholesterol as $174.9,196.0,187.5$ and $193.5 \mathrm{mg} /$ $\mathrm{dl}$, respectively. The levels of low density lipoprotein cholesterol were $106.2,127.6,122.6$ and $119.2 \mathrm{mg} / \mathrm{dl}$. Triglycerides levels were $125.6,144.5,130.1$ and $158.7 \mathrm{mg} / \mathrm{dl}$. Hence, it was concluded from the study that there was a high prevalence of various forms of lipoprotein abnormalities in Indian urban subjects (Gupta et al., 2008).

Estari et al. (2009) investigated serum lipids and the prevalence of dyslipidemia in urban adult population in Warangal district of Andhra Pradesh. A total of 1496 individuals of age group 20-29 years of age were included in the study. The results of the study indicated that $52.7 \%$ of males and $42.9 \%$ of females had at least one abnormal lipid concentration. High density lipoprotein cholesterol was abnormally low in $7 \%$ of males and in $1.6 \%$ of females. The prevalence of hypercholesterolemia, Hypertriglyceridemia and low levels of high density lipoprotein cholesterol were found to be higher in all age groups.

A cross sectional community based study was conducted in an urban community in Tenali, Andhrapradesh between July 2009 and October 2009 to assess the prevalence and risk factors for CAD by Murthy et al., 2012. A total of 534 subjects of age $\geq 20$ years were investigated. The overall prevalence rate of CAD was $5.4 \%$. Out of 29 cases of CAD, 11 cases were found to have dyslipidemia.

A study was conducted by The Indian Council of Medical Research, India Diabetes (ICMR-INDIAB) in the representative population of three states of India (Tamil Nadu, Maharashtra and Jharkhand) and one union territory (Chandigarh). The study included a total of 16,607 people of age group $\geq 20$ years using stratified multistage sampling design. The findings of the study suggested that out of the subjects studied, $13.9 \%$ had hypercholesterolemia, $29.5 \%$ had hyper-triglyceridemia, $72.3 \%$ had low high density lipoprotein cholesterol, $11.8 \%$ had high low density lipoprotein cholesterol levels and $79 \%$ had abnormalities in one of the lipid 
parameters. Regional disparity existed with the highest rates of hypercholesterolemia observed in Tamil Nadu (18.3\%), highest rates of hypertriglyceridemia in Chandigarh $(38.6 \%)$, highest rates of low high density lipoprotein cholesterol in Jharkhand (76.8\%) and highest rates of high low density lipoprotein cholesterol in Tamil Nadu (15.8\%). Low high density lipoprotein cholesterol was the most common lipid abnormality $(72.3 \%)$ in all the four regions studied. It was observed that the risk factors for dyslipidemia are obesity, diabetes and dysglycaemia (Joshi et al., 2014).

To determine the levels of cholesterol lipoproteins and prevalence of dyslipidemia in urban Asian Indians. Guptha et al., 2014 performed a study at eleven cities located in all regions of India. A total of 6123 subjects were reviewed. The cities were in Northern (Jammu, Chandigarh, Karnal, Bikaner), Western (Ahmadabad, Jaipur), Eastern (Lucknow, Patna, Dibrugarh), Southern (Madurai, Hyderabad, Belgaum) and Central(Indore, Nagpur) regions of India. The data revealed that the age adjusted prevalence in men and women were, total cholesterol $25.1 \%$ and $24.9 \%$, LDL-cholesterol $16.3 \%$ and $15.1 \%$ and HDL-cholesterol $33.6 \%$ and $52.8 \%$ and triglycerides were $42.1 \%$ and $32.9 \%$, respectively. It was concluded from the study that cholesterol level was significantly greater in subjects with better economic status, body mass index and waist circumference while triglycerides were found to be more among those with high socioeconomic status, fat intake, body mass index and waist circumference.

A study was carried out by Gupta et al., 2009 in two North Indian cities, Jaipur and Delhi to determine the prevalence of various cardiovascular risk factors in adolescents and young adults. A total of 2051 subjects (male 1009, female 1042) of age group 15-39 years were selected for the study. The results of the study indicated that high LDL cholesterol was observed in $9.4 \%$ and $8.9 \%$ in males and females, respectively. Whereas low HDL cholesterol was observed in $16.2 \%$ and $49.7 \%$, hypertriglyceridemia was observed in $9.7 \%$ and $6 \%$ in males and females, respectively. It was summarized from the study that there was low prevalence of multiple cardiovascular risk factors (smoking, hypertension, dyslipidemia, diabetes and metabolic syndrome) in adolescents and rapid escalation of these risk factors by age of 30-39 years was noted in urban Asian Indians.

One more study demonstrated the high prevalence of dyslipidemia in young Indian population (Sawant et al., 2008). A total of 1805 subjects (1128 males and 677 females) subjects of age $\geq 40$ years were selected from P.D.Hinduja Hospital, Mumbai, India. The prevalence of dyslipidemia was observed to be higher in males then in females. High level of total cholesterol was found in $38.7 \%$ males and $23.3 \%$ in females. High density lipoprotein cholesterol was abnormally low in $64.2 \%$ males and $33.8 \%$ in females. The increase of prevalence of hypercholesterolemia and hypertriglyceridemia was more prominent in 31-40 years of age group than in $\leq 30$ years of age group. It was concluded from the study that the low percentage of adults with controlled lipid concentrations suggests that there is a need for awareness programs for the prevention and control of dyslipidemia.

\section{Chemical composition of flax seed}

Flax (Linum usitatissimum) is an annual plant of the linaceae family. This plant grows to a height up to $60 \mathrm{~cm}$, with slender and very fibrous stems. The fruit contains a seed known as flaxseed or linseed (Pradhan et al., 2010). Flax is considered a functional food or source of functional ingredients, because it contains alpha-linolenic acid (Bozan and Temelli, 2008), lignans and polysaccharides, all of which have positive effects in disease prevention. The seed contains approximately 40\% lipids, 30\% dietary fiber and $20 \%$ protein.

The principal component of flaxseed is its oil, $39 \mathrm{~g} 100 \mathrm{~g}$, Cotyledons are the tissue in which oil is mainly stored, containing the highly sought-after $\alpha$-linolenic, linoleic and oleic acids. Flaxseed oil is mainly found as triacylglycerols $(98 \%)$ with lower contents of phospholipids $(0.9 \%)$ and free fatty acids $(0.1 \%)$ (Mueller et al., 2010). The average protein content in flaxseed is $22 \mathrm{~g} 100 \mathrm{~g}$ of seed. In flax, globulins are the main proteins; they make up $18.6 \%$ of the total protein, while albumin content represents $17.7 \%$ of the total protein. Flax protein is relatively rich in arginine, aspartic acid and glutamic acid, and the limiting amino acids are lysine, methionine and cysteine (Chung et al., 2005). Total fiber accounts for about $28 \%$ of the weight of full-fat flax seeds. Flax contains both soluble and insoluble dietary fiber. Dietary fiber acts as a bulking agent in the gut.

One of the most interesting characteristics of flaxseed is its content of complex phenols, such as lignans. The most remarkable one is secoisolariciresinol (SDG), although isolariciresinol, pinoresinol, mataresinol and other derivatives of ferulic acid are also present (Daun et al., 2003). Phenolics are plant derived compounds that have many different functions. Flax contains at least three types of phenolics as phenolic acids (about 1\%), flavonoids (35-70 mg/ $100 \mathrm{~g}$ ) and lignans. Lignans are found in amounts ranging from 1 $\mathrm{mg} / \mathrm{g}$ of seed to nearly $26 \mathrm{mg} / \mathrm{g}$ of seed (Muir, 2006). Flax contains 75 to 100 times more lignans than any other plant source. Flaxseed contains several water and fat-soluble vitamins as ascorbic acid thiamin, riboflavin, niacin, pyridoxine, pantothenic acid, folic acid and biotin. Flax seed also contain minerals as calcium, copper, iron, magnesium, manganese, phosphorus, potassium, sodium and zinc.

\section{Role of flax seed in management of dyslipidemia}

A recent study by Bolla and Santhi, 2016 showed significant reduction in the lipid profile by the supplementation of flax seeds for a period of 3 months. A total of 75 dyslipidemic subjects of age group 40-60 years were recruited and advised to take 25 gram of flax seeds in their diets for 3 months. It was found from the data that the mean reduction in body weight was $2.89 \%$ and the mean difference in the values of total cholesterol, triglycerides, HDL, LDL and VLDL were 16.19\%, 13.9\%, 4.94\%, 19.93\% and $17.94 \%$, respectively. Regular intake of flax seed also brought about a significant fall in the blood pressure.

Saxena and Katare (2014) had reported the therapeutic potential 
of flax seeds in mitigation of dyslipidemia. The study included 50 dyslipidemic subjects. Subjects were divided into two group as control group and experimental groups. Subject in the experimental group received $30 \mathrm{~g}$ of roasted flax seeds powder for 3 months. After supplementation there was remarkable improvement in anthropometric measurement, blood pressure and lipid profile in the experimental group. Body weight and body index of the experimental were significantly reduced. A lowering of systolic and diastolic blood pressure was also recorded in the dyslipidemic subject. A highly significant reduction in total cholesterol, triglycerides, low density lipoprotein cholesterol and very low density lipoprotein levels simultaneous elevation in high density lipoprotein cholesterol was observed. Improvement in lipid profile resulted in reduction of atherogenic indices. Therefore, it was summarized from the study that the supplementation of roasted flax seed powder for three months improved the body mass index, blood pressure and lipid profile of dyslipidemic subjects, thus exhibiting cardio protective effect.

Flax seeds are a rich source of unsaturated fatty acids, antioxidants and fibres, known to have anti-atherogenic activities. Hence, a study was conducted by Katare and Saxena, (2013) to evaluate the effect of flax seeds on serum lipid profile of dyslipidaemic patients. A total of 75 patients were selected for the study and patients were divided into 3 groups each comprising of 25 patients. Patients of group E-I were administered roasted flaxseeds chutney powder (RFCP) $30 \mathrm{~g}$ and group E- II were administered to soya nuts (SN) $30 \mathrm{~g}$ for twelve weeks. The control group was not provided with any supplementation. The results indicated that control group exhibited mean lowering of $1.81 \%$ in total serum cholesterol level followed by $16.19 \%$ and $3.79 \%$ drop in group E1 and E2. A highly significant reduction in triglyceride levels was noted in groups E-I and E-II with a mean lowering of $13.99 \%$ and $11.73 \%$, respectively. Elevation of $4.94 \%$ and $3.21 \%$ in high density lipoprotein cholesterol level was seen. Low density lipoprotein cholesterol values were also significantly lowered in group E-I and E-II with a mean change of $19.93 \%$ and $3.92 \%$. It was abstracted from the study that 12 weeks supplementation with roasted flax seed chutney powder brought about a remarkable improvement in anti-atherogenicity indices (Katare and Saxena, 2013).

Extensive studies have indicated that nutritional therapy plays a pivot role in the controlling of many diseases. Several functional foods have been shown to possess hypolipidemic properties. Flax seed (FS) is a functional food that is rich in omega 3 fatty acids and antioxidants and is low in carbohydrates. So a study on the effect of flax seed powder supplementation in the management of dyslipidemia was conducted by Mani et al., (2011). Twenty nine subjects were included in the study. Subjects were assigned to the experimental group $(n=18)$ and the control group $(n=11)$. The experimental group's diet was supplemented daily with $10 \mathrm{~g}$ of flax seed powder for a period of 1 month. The control group received no supplementation. A favorable reduction in total cholesterol $(14.3 \%)$, triglyceride $(17.5 \%)$, low density lipoprotein cholesterol $(21.8 \%)$ increased high density lipoprotein cholesterol (11.9\%) were noticed. The observation of the study indicated the therapeutic potential of flax seeds in the management of dyslipidemia.

One another study was conducted by Kristensen et al., 2012 to examine the effect of flax seed dietary fiber in different food matrics on blood lipids among 17 subjects. Three different diets were tested-a low fiber control diet (control group), a diet with flax seed fiber $\operatorname{drink}(3$ drinks/day) and a diet with flax seed fiber bread(3day). The results indicated that flax drink lowered fasting total cholesterol and LDL cholesterol by $12 \%$ and $15 \%$, respectively. Flax bread also lowered total cholesterol and LDL cholesterol by $7 \%$ and $9 \%$, respectively. Viscous flax seed fiber may be a useful tool for lowering blood cholesterol.

A study on flax seed powder suggested statistically significant reduction in blood glucose levels and blood lipid profile. The processed flax seed powder was incorporated at levels of 5\%, $10 \%$ and $15 \%$ in standardized recipes such as idli powder, bread, sesame powder boli (south Indian sweet dish) and biscuits. A total of 50 type II diabetic subjects were selected for supplementation and divided into two groups of 25 subjects each. Experimental group was supplemented with $5 \mathrm{~g}$ of flax seeds incorporated bread for a period of 90 days as evening snack. The findings showed that the mean values for lipid profile were decreased from 224.3 to $167.5 \mathrm{mg} / \mathrm{dl}$ for total cholesterol, from 178.8 to $150.9 \mathrm{mg} / \mathrm{dl}$ for serum triglyceride from 157.2 to $128.8 \mathrm{mg} / \mathrm{dl}$ for low density lipoprotein cholesterol and the mean values increased significantly from 36.5 to $52.1 \mathrm{mg} / \mathrm{dl}$ for high-density lipo-protein cholesterol. The findings had revealed that the use of flax seed powder had immense therapeutic potential (Nazni et al., 2006).

\section{Role of Flax Seed Oil in Management of dyslipidemia-}

Flax seed oil is mainly considered as a healthy food. Fatty acid composition of regular flax seed oil is different from other commercial oils because of the very good composition of, alpha linolenic acid usually above $50 \%$. To compare the effect of hempseed oil (HO) and flax seed oil (FO) on the profile of serum lipids and fasting concentration of serum total and lipoprotein lipids, plasma glucose and insulin in healthy humans. Schwab et al. (2006) had conducted a study. Fourteen healthy volunteers participated in the study. The subjects were asked to consume hempseed oil and flax seed oil $30 \mathrm{ml} /$ day for 21 weeks each. The periods were separated by a four week wash-out period. The data revealed that the hempseed oil period resulted in a lower total of HDL cholesterol ratio compared with the flax seed oil period $(p=0.065)$. The effect of $\mathrm{HO}$ and FO on the profile of serum lipid was found to be different significantly, with only minor effects on concentration of fasting serum total or lipoprotein lipids, and no significant changes in concentration of plasma glucose or insulin (Schwab et al., 2006).

Alpha linolenic acid (ALA) is the natural precursor of cardio protective long chain $n=3$ fatty acids. Paschos et al. (2007) had conducted a study to examine the effect of increased ALA intake on blood pressure in middle aged dyslipidemic men. The dietary supplementation of flax seed oil rich in alpha linolenic acid (8 g/ 
day), examined to see the effect on blood pressure of dyslipidaemic patients. Diet of control group $(\mathrm{n}=28)$ was supplemented with safflower oil, containing the equivalent $\mathrm{n} 6$ fatty acids $(11 \mathrm{~g} /$ day $)$ linoleic acid. After 12 weeks supplementation with ALA resulted in the significantly lower systolic and diastolic blood pressure levels compared with LA ( $\mathrm{p}=0.016$ and $\mathrm{p}=0.011$, respectively). In conclusion, dietary supplementation with $8 \mathrm{~g}$ /day ALA for 12 weeks lowered both SBP and DBP in dyslipidaemic men. The magnitude of hypotensive effect $(5 \mathrm{~mm} / \mathrm{hg}$ or $3-6 \%)$ was certainly clinically relevant, and is expected to considerably reduce the overall CVD risk in the patients (Paschos et al., 2007).

Recently, novel dietary oils with modified fatty acid profiles have been manufactured to improve fatty acid intakes and to reduce cardiovascular diseases risks. A study by Gillingham et al. (2011) was conducted to evaluate the efficacy of novel high oleic rapeseed (canola) oil (HOCO), alone or blended with flax seed oil (FXCO), on circulating lipids and inflammatory biomarkers over a typical western diet (WD). A total of thirty six hypercholesterolemic subjects were included in the study and asked to consume three isoenergetic diets for 28 days. Experimental diets were designed as typical western diet contained $50 \%$ energy as carbohydrate, $15 \%$ as protein and $35 \%$ as fat, of which $70 \%$ was provided with experimental oil. The study was designed as three phases with 28 days per phase separated by $4-8$ weeks wash-out periods. Subjects consumed three isoenergetic diets. Each contained approximately $36 \%$ energy from fat, of which $70 \%$ was provided by HOCO, FXCO or WD. The results of the study indicated that the total cholesterol was reduced by $11 \%(\mathrm{p}=0.001)$ with flax seed oil and $3.5 \%$ reduction was found in HOCO group. After 28 days compared with WD, LDL cholesterol was reduced $15.1 \%$ with FXCO and $7.4 \%$ with HOCO group. In conclusion, consumption of novel HOCO alone or blended with flax seed oil is cardioprotective through lipid lowering effects (Gillingham et al., 2011).

Kawakami et al. (2015) investigated the effect of 12 week supplementation with flax seed oil (FO), which is a rich source of alpha linolenic acid (ALA), on cardiovascular risk factors such as serum small dense low-density lipoprotein concentration. A total of 15 subjects were included in the study. The subjects were instructed to take $10 \mathrm{~g}$ of flax seed oil or corn oil for 12 weeks using spoon provided, once with dinner. Supplements of $10 \mathrm{~g}$ of flax seed oil and corn oil contained 5.49 and $0.09 \mathrm{~g}$ of ALA, respectively. Subjects were instructed to maintain their habitual diet throughout the study. Subjects were also instructed to avoid intake of anti-inflammatory drugs vitamins or other dietary supplements throughout the intervention period. The data revealed that the flax seed oil supplementation reduced low density lipoprotein cholesterol concentration by $25.8 \%$ and $21.2 \%$ at 4 and 12 weeks, respectively. Although, corn oil supplementation had no effect on LDL concentrations. It was also abstracted from the study that serum total cholesterol, LDL-cholesterol, HDL-cholesterol were significantly lowered in the flax seed oil supplementation (Kawakami et al., 2015).

International Journal of Nutritional Science and Food Technology An open Access Journal

\section{References}

1. Bloedon, L., T., Szapary, P., O. (2004). Flax seed and cardiovascular risk. Nutr Rev, 62, 18-27.

2. Bolla, K., Santhi, K.V. (2016). Impact of flax seed consumption on dyslipidemia. Food Science and Quality Management, ISSN, 48.

3. Bozan, B., Temelli, F. (2008). Chemical composition and oxidative stability of flax, safflower and poppy seed and seed oils. Bioresour Technol, 99, 6354-6359.

4. Chartlet, P., Hongsprabhas, P., Pisprasert, V., Pongchayakul, C. (2006). Rural urban differences in lipid profile and prevalence of dyslipidemia: A population based study in Khon Kaen province, Thailand. J Med Assoc Thai, 89(11): 1835-1844.

5. Chaturvedi, V., Bhargava, B. (2007). Health care delivery for coronary heart disease in India-Where are we headed. Am Heart Hosp J, 5, 32-37.

6. Chung, M., Lei, B., Chan, L. E. (2005). Isolation and structural characterization of the major protein fraction from norman flax seed (Linum Usitatissium L.). Food Chem, 90, 271-279.

7. Daun, J., Barthet, V., Chornick, T., Duguid, S. (2003). Structure, composition and variety development of flax seed. Flax seed in Human Nutrion, 2nd edition Champaign, Illinois, 1-40.

8. Estari, M., Reddy, A. S., Bikshapathi, A., Satyanarayana, J., Venkanna, L., Reddy, M. K. (2009).The investigation of serum lipids and prevalence of dyslipidemia in urban adult population of Warangal district, Andhra Pradesh. India Biology and medicine, 1(2): 61-65.

9. Gillingham, L. G., Gustafson, J. A., Han, S. Y., Jassal, D. S., Jones, P. J. H. (2011). High-oleic rapeseed (Canola) and flax seed oils modulate serum lipids and inflammatory biomarkers in hypercholesterolemic subjects. British Journal of Nutrition, 105, 417-427.

10. Gupta, R., Gupta, S., Agarwal, A., Kaul, V., Gaur, K., Gupta, V. P. (2008). Secular trends in cholesterol, lipoprotein and triglycerides and prevalence of dyslipidemiain an urban Indian population. Lipid in Health and Disease, 40(7): 1476-1511.

11. Gupta, R., Misra, A., Vikram, N. K., Kondal, D., Gupta, S. S., Agarwal, A., Pandey, R.M. (2009). Younger age of escalation of cardiovascular risk factors in Asian Indian Subjects. BMC Cardiovascular Disorders, $9(28)$.

12. Guptha, S., Gupta, R., Deedwania, P., Bhansali, A., Maheshwari, A., Gupta, A., Gupta, B., Singh, J., Achari, V., Sharma, K.K. (2014). Cholesterol lipoproteins and prevalence of dyslipidemia in urban Asian Indians: A cross sectional study. Indian Heart Journal, $66,280-288$.

13. Indrayan, A. (2005). Forecasting vascular disease cases and associated mortality in India. Reports of the National Commission 
on Macroeconomics and Health Ministry of Health and Family Welfare, India, http://www.whoindia.org/EN/Section102/Section 201-888.htm.

14. Joshi, S. R., Anjana, R. M., Deepa, M., Pradeepa, M., Bhansali, A., Dhandania, V. K., Joshi, P. P., Unnikrishnan, R., Nirmal, E., Subhashini, R. K., Madhu, S. V., Rao, P. V., Das, A. K., Kaur, T., Shukla, D. K., Mohan, V. (2014). Prevalence of dyslipidemiain urban and rural India. Plos One, 9(5): e96808.

15. Katare, C., Saxena, S. (2013). Amelioration of selected cardiac risk factors through supplementation of diet with flax seed and soyabeen. International Journal of Nutrition, Pharmacolgy, Neurological Diseases, 3, 4.

16. Kawakami, Y., Okumura, H. Y., Kuroki, Y. N., Taketan, Y., Takeda, E. (2015). Flax seed oil intake reduces serum small dense low density lipoprotein concentrations in Japanese men: a randomized double blind, cross-over study. Nutrition Journal, 14, 39.

17. Kristense, M., Jensen, M. G., Aarestrup, J., Peterson, E. N., Sonderguard, L., Milkkensen, M. S., Astrup, A. (2012). Flax seed dietary fibers lower cholesterol and increase fecal fat excretion, but magnitude of effect depends on food type. Nutrition and Metabolism, 9(8).

18. Lee, M. H., Kim, H. C., Ahn, S. V., Hur, N. W., Choi, D. P., Park, C. G., Suh, I. (2012). Prevalence of dyslipidemia among Korean adults: Korea National Health and Nutrition Survey 19982005. Diabetes Metab J, 36,43-55.

19. Mani, U. V., Mani, I., Biswas, M., Kumar, S. N. (2011). An open label study on the effect of flax seed powder (Linum Usitatissimum) supplementation in the management of diabetes mellitus. Journal of Dietary Supplements, 8(3): 257-265.

20. Margaret, M., Hertz, R. P., Unger, A. N., Lustik, M. B. (2009). Prevalence, awareness and management of hypertension, dyslipidemia and diabetes among United States adults aged 65 and older. J Gerontol A Biol Sci Med Sci, 64, 64(2), 256-263.

21. Mishra, A., Pandey, R. M., Ramadevi, J., Sharma, R., Vikram, N. K., Khanna, N. (2001). High prevalence of diabetes, obesity and dyslipidemiain urban slum population in Northern India. International Journal of Obesity, 25(11): 1722-1729.

22. Mueller, K., Eisner, P., Stark, Y. Y., Nakada, R., Kirchhaff, E. (2010). Functional properties and chemical composition of fractionated brown and yellow linseed meal (Linum Usitatissimum). J Food Eng, 98(4): 453-460.

23. Muir, A. D. (2006). Flax lignans- analytical methods and how they influence our understanding of biological activity. JAOAC Int, 89, 1147-1157.

24. Murthy, P. D., Prasad, K. T., Gopal, P. V., Rao, K. V., Rao, R. M. (2012). A survey for prevalence of coronary artery disease and its risk factors in an urban population in Andhra Pradesh, JAPI, 60.

25. National cholesterol education programme (NCEP), Expert panel on Detection, evaluation and treatment of high blood cholesterol in adults (Adult treatment panel III), Final report. (2002). National Institute of Health, Pub. No. 02-5215.

26. Nazni, P., Amrithaveni, M., Kumar, V. P. (2006). Impact of flax seeds based therapeutic food on selected type II diabetic patients. Ind J Nutri Dietet, 43,141.

27. Paschos, G. K., Magkas, F., Panagiotakos, D. B., Votteas, v., Zampelas, A. (2007). Dietary supplementation with flax seed oil lowers blood pressure in dyslipidemic patients. European Journal of Clinical Nutrition, 61, 1201-1206.

28. Pradhan, R., Meda, V., Rout, P., Naik, S., Datai, A. (2010). Supercritical $\mathrm{Co} 2$ extraction of fatty oil from flaxseed and comparison with screw press expression and solvent extraction processes. J Food Eng, 98(4), 393-397.

29. Sawant, A. M., Shetty, D., Mankeshwar, R., Ashavaid, T. F. (2008). Prevalence of dyslipidemiain young adult Indian population. JAPI, 56.

30. Saxena, S., Katare, C. (2014). Evaluation of flax seed formulation as a potential therapeutic agent in mitigation of dyslipidemia, Biomed J, 37(6): 386-90.

31. Schwab, U. S., Callaway, J. C., Erkkila, A. T., Gynther, J., Uusitupa, M. I. J., Jarvinen, T. (2006). Effects of hempseed and flax seed oils on the profile of serum lipids, serum total and lipoprotein lipid concentrations and haemostatic factors. http:// link.springer.com

32. Thompson, L., Boucher, B., Liu, Z., Catterchio, M., Krieger, N. (2006). Phytoestrogen content of foods consumed in Canada, including isoflavones, lignans and caumestan. Nutrition and Cancer, 54(2), 184-201.

33. Wang, S., Liang, X. U., Jast, B., You, J. S., Wang, Y. Z., Yang. (2011). High Prevalence and associated factors of dyslipidemiain the adult chinese population. Plos ONE, 6, I-3, e17326.

34. Wong, N. D., Lopez, V., Simon, T., Williams, G. R. (2006). Prevalence, treatment and control of combined hypertension and hypercholesterolemia in the United States. The American Journal of Cardiology, 98(2): 204-208.

35. Zhao, W. H., Zhang, J., Zhai, Y., Man, Q. Q., Wang, C. R., Li, H., Li, Y., Yang, X. G. (2007). Blood lipid profile and prevalence of dyslipidemia in Chinese adults. Biomedical and Environmental Sciences, 20(4), 329-335.

36. Zhiyan, L. I., Yang, R., Guabing, X. U, Yia, T. (2005). Serum lipid concentrations and prevalence of dyslipidemiain a large professional population in Beizing. Clinical Chemistry, 5(11): 144-150. 